\title{
A modified genetic algorithm for time and cost optimization of an additive manufacturing single-machine scheduling
}

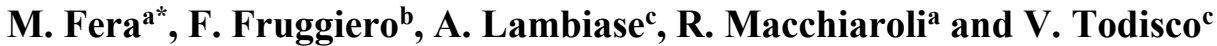

a Department of Industrial and Information Engineering, University of Campania "Luigi Vanvitelli", Via Roma 29, Aversa, CE, Italy

${ }^{b}$ University of Basilicata, School of Engineering, Via Nazario Sauro, 85, Potenza, PZ, Italy

${ }^{c}$ Department of Industrial Engineering, University of Salerno, Via Giovanni Paolo II, Fisciano, SA, Italy

\section{CH R O N I C L E}

\section{Article history:}

Received October 202017

Received in Revised Format

December 252017

Accepted January 142018

Available online

January 162018

Keywords:

Additive Manufacturing

Scheduling

Time

Cost

Metaheuristics

Production Planning \begin{abstract}
A B S T R A C T
Additive Manufacturing (AM) is a process of joining materials to make objects from 3D model data, usually layer by layer, as opposed to subtractive manufacturing methodologies. Selective Laser Melting, commercially known as Direct Metal Laser Sintering (DMLS ${ }^{2}$ ), is the most diffused additive process in today's manufacturing industry. Introduction of a DMLS $®$ machine in a production department has remarkable effects not only on industrial design but also on production planning, for example, on machine scheduling. Scheduling for a traditional single machine can employ consolidated models. Scheduling of an AM machine presents new issues because it must consider the capability of producing different geometries, simultaneously. The aim of this paper is to provide a mathematical model for an AM/SLM machine scheduling. The complexity of the model is NP-HARD, so possible solutions must be found by metaheuristic algorithms, e.g., Genetic Algorithms. Genetic Algorithms solve sequential optimization problems by handling vectors; in the present paper, we must modify them to handle a matrix. The effectiveness of the proposed algorithms will be tested on a test case formed by a 30 Part Number production plan with a high variability in complexity, distinct due dates and low production volumes.
\end{abstract}

\section{Introduction}

Additive Manufacturing (AM) is a topic that is experiencing a continuous enlargement. In fact, it collects an increasing number of researches year by year and covers several research areas, from the design of the products and material investigations to manufacturing issues. This paper aims to analyze how to schedule production orders for a single AM (DMLS ${ }^{\circledR}$ ) machine by considering a time and cost optimization framework. The purpose is to find a mathematical model that is useful for production planners who must schedule an AM production that is both time- and cost-efficient, perfectly in line with Lean Manufacturing principles.

Section 2 presents a formalization of the problem that will be studied in this paper. Section 3 presents a brief literature review about AM in an actual specified research field: production planning. The literature

* Corresponding author

E-mail: marcello.fera@unicampania.it (M. Fera)

2018 Growing Science Ltd.

doi: $10.5267 /$ j. ijiec.2018.1.001 
is systematically reviewed, but not all suggestions seem to be aligned with the actual needs of the industrial sector for a single-machine AM machine Scheduling Problem (AMSP). Section 4 presents the model formulation for AMSP, with a multi-objective function (OF) that is subject to constraints on the geometrical volume of the parts to be produced and on other production variables. In this section, the OF combinatorial Optimization Problem (MOP) is divided into time and cost parts, each one is represented in detail, and the computational complexity for AMSP is demonstrated to be NP-HARD. Next, Section 5 provides a solution to AMSP using a traditional metaheuristic algorithm like Genetic Algorithms (GAs). It is worth noting that this algorithm alone cannot provide a solution to AMSP. The GA needs some changes to operate over the proposed mathematical model. A GA based on a 2D crossover will be presented in details. In Section 6, a test case for checking the effectiveness of the proposed algorithm is presented, by considering time and cost reduction, and also the running time. In Section 7, new ideas for future improvements and researches are proposed.

\section{Open issue/scheduling problem analysis}

A traditional scheduling problem is defined as follows:

"Given a set of jobs $J=\left\{J_{1}, J_{2}, J_{3}, \ldots\right\}$ and a set of machines $M=\left\{M_{1}, M_{2}, M_{3}, \ldots\right\}$, assign job to resources to optimize (minimize/maximize) an objective (certain goal)."

The production systems that satisfy the demands for orders or for the stocks generally produce parts dividing the demand in smaller parts, which are defined as batches of a specific part number (PN). When we need to pass from a PN to another, a change is needed. Batch quantity is accurately chosen to minimize the setup number during the production, since this is an activity without added value.

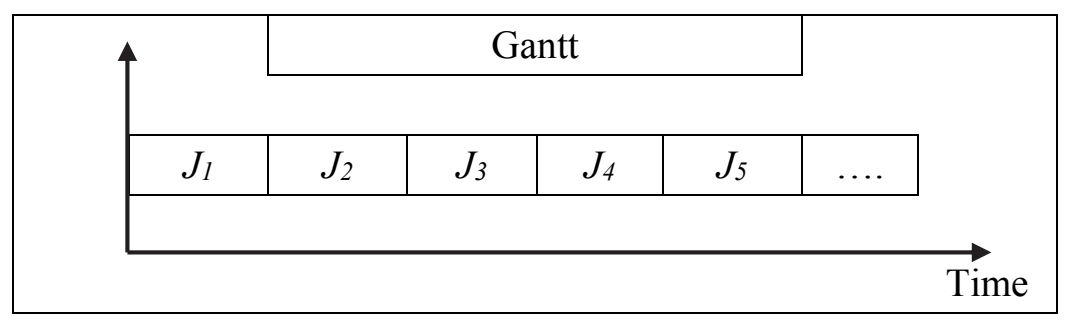

Fig. 1. Traditional schedule shape

The scheduling problems are commonly represented using a Gantt diagram (Fig. 1), which shows how a single-machine scheduling problem becomes a sequential optimization problem, where sequence is a vector of jobs. In fact, the goal is to find the best combination of jobs on the machine to optimize a certain objective: lateness, tardiness, flow time, number of late job, make span, etc.

Process sequence in computational terms is a vector resumed as follows:

$$
S_{S M}=\left[J_{1}, J_{2}, J_{3}, \ldots\right] \rightarrow S_{S M}=[1,2,3 \ldots] .
$$

The aim of traditional single-machine scheduling is to find the best combination of quantities to be produced in advance, i.e., jobs.

Scheduling principles change in case of AM machines. A generic $j$-th job can be constituted by several geometries. The job is now heterogeneous in AM, i.e., the single production run can involve several PNs and not only one like in traditional machines. This news can be summarized as follows. Let us denote "build" as a set of several traditional jobs that can be identified as the couple constituted by $G$ and $n$, where $G$ identifies the geometry type to be produced and $n$ is the number of parts to be produced for the $j$-th production run called build.

$$
\text { build }_{j} \leftrightarrow\left\{\left(G_{1}, n_{1}\right),\left(G_{2}, n_{2}\right),\left(G_{3}, n_{3}\right), \ldots,\left(G_{i}, n_{i}\right)\right\}
$$


Job is a heterogeneous concept in AM, corresponding with build chamber composition, usually known synthetically as build. To point out this latter concept, we can propose a figure by Baumers.

A build is made up of various PNs/Geometries to produce each one with its independent number $n_{i, j}$, as it is possible to see from a representation of a generic build for an AM machine.

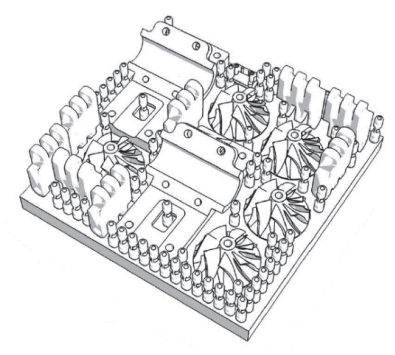

Fig. 2. Build concept

$$
\text { build }_{j}=\left[n_{1}, n_{2}, \ldots, n_{n_{g}}\right] \text {. }
$$

It is worth noting that there is a possibility that a single build could not satisfy the overall demand, so the planner must divide production in several builds. Index $j$ for the builds goes from 1 to $n_{b}$, i.e., the required number of build needed to complete production. Therefore, the AM machine schedule form is a set of builds, i.e., a matrix, as shown in Table 1.

The AM scheduling problem is summarized in the following research questions:

1. What is the number of each geometry/PN for each build?

2. How many builds are necessary to complete production?

The research questions for AM are different from the ones for SM. To introduce the research questions on AM scheduling, literature review is presented, which will review internationally published papers on single-machine scheduling, AM or SM, with similarities to the research questions previously introduced.

Table 1

AM schedule model shape

\begin{tabular}{|c|c|c|c|c|c|c|}
\hline \multirow{2}{*}{$S$} & & \multicolumn{5}{|c|}{ Geometries } \\
\hline & & 1 & 2 & 3 & $\ldots$ & $n_{g}$ \\
\hline \multirow{5}{*}{ 鸪 } & 1 & $n_{1,1}$ & $n_{2,1}$ & $n_{3,1}$ & $\ldots$ & $n_{g, 1}$ \\
\hline & 2 & $n_{1,2}$ & $n_{2,2}$ & $n_{3,2}$ & $\ldots$ & $n_{g, 2}$ \\
\hline & 3 & $n_{1,3}$ & $n_{2,3}$ & $n_{3,3}$ & $\ldots$ & $n_{g, 3}$ \\
\hline & $\ldots$ & $\ldots$ & $\ldots$ & $\ldots$ & $\ldots$ & $\ldots$ \\
\hline & $n_{b}$ & $n_{n b, 1}$ & $n_{n b, 1}$ & $n_{n b, 3}$ & $\ldots$ & $n_{n b, n g}$ \\
\hline
\end{tabular}

\section{Literature review}

In the last 20 years, papers about AM have been increased systematically. According to Witherell et al. (2017), Costabile et al. (2017), Fera et al. (2016a), and Fera et al. (2017), this is a multi-disciplinary topic because it links together design, material science, energy consumptions, life cycle management, laser technology, computer science, supply chain management, and production planning. The importance of the AM research field of management is witnessed also by Pour et al. (2016), who presented a set of proposals to reconfigure the production system and supply chain to enable AM as a reliable and functional system. The importance of the AM is also witnessed by the use of this technology in terms of the interaction between the machines and the humans as underlined by Fruggiero et al., 2016 and the use 
of this new technology in terms of $\mathrm{CO}_{2}$ emissions reduction during the life span as underlined by Fera et al. (2016b). The importance of the AM in the production context is witnessed also by the fact that this technology is one of the enabling technologies for the achievement of the Industry 4.0 paradigms (Dilberoglu et al., 2017; Fruggiero et al., 2016).

Over the years, several papers on all of the above research fields have been published, but very few seem to consider an AM machine in the production department context, maybe because the first studies were devoted more to understanding the capability of this new technology, especially using metals instead of plastic powders. This paper aims to analyze how to schedule production orders for an AM (DMLS®) machine to achieve time and cost optimization.

A systematic literature review was made to discover possible sources for the proposed paper goal, i.e., AM scheduling problem. Unfortunately, the sources are not so many, but few of them are, however, present in the cited databases. The theme of the process planning using AM is known as a research theme since 2008 (Ren et al., 2008), but it was not developed for the reasons about the reliability of the production process in terms of mechanical properties of the metal products.

The first source is Li et al. (2017), a work about production planning of distributed AM machines to fulfil demands received from individual customers in low quantities. The aim of the paper is to understand how to group the given parts from different customers and how to allocate them to various machines to minimize average production cost per volume of raw material. The authors recognized that the problem is not resolvable in acceptable time by a normal CPU, so they preferred to create two different heuristics. The heuristics take into account the fact that AM machines are different, located in several parts of the globe, and two main data for the products to be realized are available, i.e., maximum part height and production area of the machine. It is worth noting that this is a good way to optimize the problem, but it neglects the important fact that, sometimes with support structures, the machine chamber allows a part on top of each other. Moreover, the aim of this paper is to investigate the scheduling of a single machine in a specific production system, not in a geographically distributed environment, so that the paper can give some advice on the problem, such as the complexity and the mathematical model, but it is a different problem from the one discussed in this paper.

Ransikarbum et al. (2017) proposed a decision support model based on a multi-objective optimization for orientation of a batch of parts and multiple printers, given fixed, un-rotated orientation of parts. A model that considers operating cost, load balance among printers, total tardiness, and total number of unprinted parts as objectives in the fused deposition modelling process is provided. Even if this model is close to the answer to our research question, for the fact that it refers to a multi-printer distributed environment, it does not fulfil the objective of the present research. Jin et al. (2017) focused their attention on the process planning theme using AM. The research dealt with the definition of a process planning to minimize the raw material consumption for the AM. Another interesting work about the assignment of a specific job to a build was presented by Zhang et al. (2016). This work focused on how to optimally place multi-parts onto the machine build platform or in build space with respect to user-defined objectives. The authors presented this problem as an NP-HARD 3D space problem, being a variant of nesting or packaging problem in 2D. The method is based on a two-step algorithm: the first step is to choose the part's orientation, and the second step defines the assignment of the part with an orientation to a specific build. Other sources available on related research themes close to the one investigated in this paper are about the use of the process planning techniques for the re-working processes using AM or combining AM with the subtractive technologies. $\mathrm{Zu}$ et al. (2017), starting from a framework defined by Newman et al. (2015) investigated specifically this research theme by defining a new decision support model to combine the old and the new production technologies in terms of process planning with the objective to optimize the modification process of existing products. 
As it is possible to see from the previous literature review, the valuable sources are not really focused on the research theme, purpose of this paper. To summarize, all reviewed models tried to consider AM machines in the production planning context, also in interaction with the traditional production machines, but no details on single-machine scheduling were presented. The main part of the sources was on the process planning and not on the scheduling of the single AM machine. Specifically, there are no answers to our two research questions at the end of Section 2. Therefore, the research questions in this paper are open issues in the research field introduced so far.

\section{Model formulation}

As introduced so far, AM scheduling is a problem to be solved since this technology started to be a permanent part of the production environment of several companies, especially in the fields of defense and aerospace. The question that the paper wants to answer is always the same for all scheduling problems, i.e.:

"What's the schedule that allows to respect due dates with the least production cost?"

The question is the same, but the context as explained in the Introduction is very different from the traditional ones for the motivations illustrated in the previous paragraphs. Therefore, let us introduce a multi-objective model for the AM scheduling that is able to consider also the new constraints given by the new context. This type of model in literature is known as Multi-Objective Optimization Problem (MOP) because it presents double objectives: time and cost.

In our vision (Fig. 3), Production orders are the inputs of the AMSP; the attributes of an order are

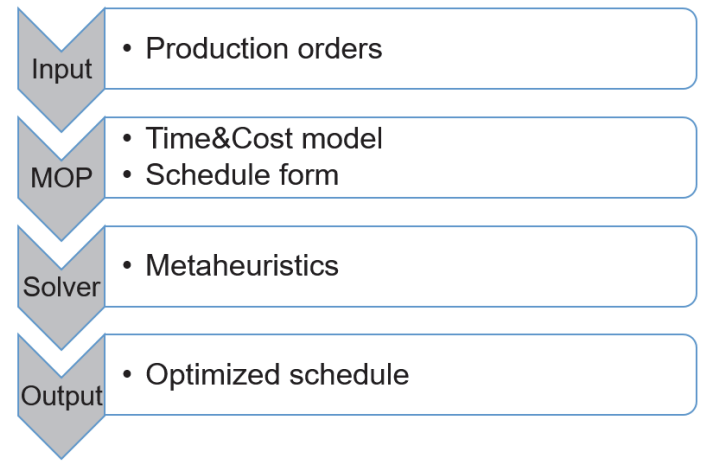

Fig. 3. Mathematical model frame

$$
\begin{array}{llr}
d_{i}: & \text { demand of } G_{i}-t h \text { geometry or PN } & \text { [part }] \\
d d_{i}: & \text { due date of } G_{i}-\text { th } \text { geometry or PN } & {[\text { day }]} \\
V_{i}: & \text { volume of } G_{i}-\text { th geometry or PN } & {\left[\mathrm{cm}^{3}\right] .}
\end{array}
$$

After that the attributes for the production orders are listed, it is worth noting that, in this paper, a Time $\&$ Cost model will be applied. In particular, the Completion Time (CT) and the Total Part Cost (TPC) will be considered. CT is the time to produce a single unit of $G_{i}-t h$ geometry, whereas TPC is the costs to be covered to produce a single part, and it is possible to compute itself using the method illustrated in Fera et al. (2017). Once the main description elements of our model are described, let us introduce the mathematical formulation of the optimization problem analyzed here. The basic model is taken from a research paper that used earliness and tardiness as objective functions (Nearchou, 2010); in this proposal, cost is added.

$\min F_{S}=F_{E T}+F_{C P}$

where 


$$
\begin{aligned}
& F_{E T}=\sum_{i}^{n_{g}}\left[\alpha_{S} E_{i}+\beta_{S} T_{i}\right] \\
& F_{C P}=\sum_{i}^{n_{g}} \gamma_{S} T O C_{i} \\
& \text { subject to: } \\
& \sum_{i}^{n g} n_{i, j} * V_{i} \leq V_{\text {available }} \forall j \in\left[1, n_{b}\right] \\
& \sum_{j}^{n b} n_{i, j}=d_{i}, \quad \forall i \in\left[1, n_{g}\right] \\
& \alpha_{S}, \beta_{S}, \gamma_{S}, T O C_{i}, V_{i}, V_{\text {available }} \in \mathbb{R}^{+} \\
& E_{i}, T_{i}, i, j, n_{g}, n_{b}, d d_{i} \in \mathbb{Z}^{+} .
\end{aligned}
$$

The proposed scheduling model has some hypotheses that are listed below:

- The scheduling problem faced here is a Single-machine scheduling problem, where the machine is an AM machine: DMLS ${ }^{\circ}$ or SLM process based.

- The part orientation is given, and the required space for manual part removal is presented.

- The build chamber allows construction of parts on top of each other by support structures or other solutions.

- Stock costs are neglected.

\subsection{Objective function}

Let us start to explain the completion time part of the objective function. The due date respect is the first goal to achieve. Production planning must balance earliness and tardiness, two concepts summed up by the term lateness.

$$
E_{j}=\max \left(0, d d_{j}-C_{j}\right), T_{j}=\max \left(C_{j}-d d_{j}, 0\right) .
$$

In the previous equations, $C_{j}$ is the completion time, and $d d_{j}$ is the due date of $j-t h$ order.

Earliness must be compressed to reduce inventory costs as stated in the JIT and Lean Manufacturing theory. Tardiness must be minimized to avoid monetary or strategic penalties. Starting from these wellknown facets, it is possible to estimate the tardiness damages as a monetary penalty proportional to each day of delay, and strategic damage will be neglected in this study because of the difficulty in evaluating and estimating it.

A common way to model the E\&T problem is

$1 \| \sum_{j=1}^{n} \alpha E_{j}+\beta T_{j}$

where

$E_{j}: \quad$ Earliness of $j-t h$ job

[day]

$T_{j}$ : $\quad$ Tardiness of $j$-th job

[day]

$n$ number of job 
$\alpha$ : constant weigh for $\mathrm{E}$

$[1 /$ day $]$

$\beta: \quad$ constant weigh for $\mathrm{T}$

$[1 /$ day $]$

$\alpha, \beta$ are constant weights computed as follows:

$\alpha=\frac{1}{\max \left(E_{j}\right)-\min \left(E_{j}\right)}, \quad \beta=\frac{1}{\max \left(T_{j}\right)-\min \left(T_{j}\right)}$.

An important concept to underline is that, in AM, E\&T of the completion time of an order is not related to the processing time on the same order (geometry). In fact, a single order can be divided into a certain number of builds, each of them with an own geometrical mix; this means that, with AM, different jobs can be performed simultaneously, achieving the number of parts due to the client in parallel with others. The classical problem of set-up is represented in a very different way, since the raw material should be ready for all of the geometries to be built or for the preparation of the building program on the machine, but all the times to change production related for example to the tools change are no more present.

The completion time of an order binds itself to the processing time of each build in which it is divided. To clarify this concept, please check the example in Table 2.

Table 2

Example of job division between different buildings

\begin{tabular}{|c|c|c|c|c|c|c|}
\hline \multirow[b]{2}{*}{$S$} & & \multicolumn{4}{|c|}{ Geometries } & \multirow[t]{2}{*}{$\mathrm{C}_{\mathrm{j}}$} \\
\hline & & 1 & 2 & 3 & 4 & \\
\hline \multirow{4}{*}{ 营 } & 1 & 7 & 3 & 2 & 0 & 18 \\
\hline & 2 & 1 & 0 & 1 & 1 & 26 \\
\hline & 3 & 0 & 4 & 0 & 0 & 34 \\
\hline & 4 & 0 & 0 & 0 & 1 & 39 \\
\hline \multicolumn{2}{|c|}{$D D i$ day] } & 25 & 30 & 26 & 11 & \\
\hline
\end{tabular}

In Table 2, order \#2 is clustered in build $_{1}$ and build $_{3}$, and it will have a completion time of 34 days, with 4 days of tardiness. Therefore, we propose a new version of the E\&T equation used in the AM context:

$$
\begin{aligned}
E_{i} & =\max \left(d d_{i}-C_{j}{ }^{i, \text { end }}, 0\right) \\
T_{i} & =\max \left(C_{j}^{i, \text { end }}-d d_{i}, 0\right)
\end{aligned}
$$

where

$E_{i}: \quad$ Earliness of $i-t h$ order

$T_{i}: \quad$ Tardiness of $i-t h$ order

$d d_{i}$ : $\quad$ due date of $i-t h$ order

$C_{j}^{i, \text { end }}$ : $\quad$ completion time of $j-t h$ build, the last in which $i$-th order has been divided.

It is worth noting that the difference between the clustering of the production order in different builds is very different from the one proposed in the past, when the order was clustered focusing on the number of set-up minimization. Therefore, in the past, clustering focused on minimizing the completion time, but maximizing the number of objects processed in a single job on the machine. Now, the objective is always the same, but the number of objects for a single build does not have to be the maximum possible with respect to the delivery dates, but the maximum that can be hosted in the build camera volume, to optimize the volume saturation of the camera, which is recognized as a key factor for AM machine optimization (Fera et al., 2017). The E\&T objective function in the case of the AMSP is modified as follows:

$F(S)_{E T}=\sum_{i=1}^{n_{g}}\left(\alpha_{S} E_{i}+\beta_{S} T_{i}\right)$ 
where

$\begin{array}{lll}\alpha_{S}: & \text { Earliness constant weight } & {[1 / \text { day }]} \\ \beta_{S}: & \text { Tardiness constant weight } & {[1 / \text { day }]} \\ E_{i}: & \text { Earliness of } i-t h \text { order } & {[\text { day }]} \\ T_{i}: & \text { Tardiness of } i-t h \text { order } & {[\text { day }]} \\ n_{g}: & \text { number of order/geometries } & {[-]} \\ F_{E T}: & \text { Time part of proposed MOP } & {[-]}\end{array}$

$\alpha, \beta$ are constant weights related to $s-t h$ schedule and they are computed as follows:

$$
\alpha_{S}=\frac{1}{\max \left(E_{S}\right)-\min \left(E_{S}\right)}, \quad \beta_{S}=\frac{1}{\max \left(T_{S}\right)-\min \left(T_{S}\right)} .
$$

The first part of MOP focuses on the balanced reduction of E\&T, so we expect a schedule where order completion dates are in a restricted neighborhood of the established due dates. As introduced before, the MOP also has a cost part (the $\boldsymbol{F}_{\boldsymbol{C P}}$ part). For this cost part, we will refer to a cost model specifically developed for the AM, recently discussed in Fera et al. (2017).

The information needed to implement the costing model are

- Unitary Completion time (CT);

- Unitary Part Cost (TPC);

- Schedule configuration as reported in Fig. 6.

The TPC model allows computing the $i-t h$ PN unit cost in whatever build it is located. In the following table, they are presented in their typical form.

Table 3

Total production cost per part

\begin{tabular}{|c|c|c|c|c|c|c|c|c|c|}
\hline \multirow{2}{*}{\multicolumn{2}{|c|}{$T P C$}} & \multicolumn{8}{|c|}{ Geometries } \\
\hline & & & 1 & & 2 & & 3 & & 4 \\
\hline \multirow{4}{*}{$\frac{\overline{0}}{\overline{\bar{c}}}$} & 1 & $€$ & 554,2 & $€$ & - & $€$ & 953,4 & $€$ & 635,1 \\
\hline & 2 & $€$ & - & $€$ & $1.231,4$ & $€$ & - & $€$ & 658,8 \\
\hline & 3 & $€$ & - & $€$ & - & $€$ & $1.156,6$ & $€$ & - \\
\hline & 4 & $€$ & - & $€$ & $1.395,4$ & $€$ & - & $€$ & 563,7 \\
\hline
\end{tabular}

Element-by-element multiplication between the schedule matrix of Table 2 and TPC, computed following the model in Fera et al. (2017) and presented in Table 3, provides a third matrix (Table 4), a sort of cost distribution along the schedule. The same geometry presents different specific costs, depending on the production mix of build where it is located. The total order cost $(O C)$ is the cost to produce an order with the clustering in a possible schedule in different builds. This OC is formally defined as follows:

Table 4

Production cost per build

\begin{tabular}{|c|c|c|c|c|c|c|c|c|c|}
\hline \multirow{2}{*}{\multicolumn{2}{|c|}{$S^{*} T P C[€]$}} & \multicolumn{8}{|c|}{ Geometries } \\
\hline & & \multicolumn{2}{|r|}{1} & \multicolumn{2}{|r|}{2} & \multicolumn{2}{|r|}{3} & \multicolumn{2}{|r|}{4} \\
\hline \multirow{4}{*}{ 음 } & 1 & $€ \quad$ & $3.879,3$ & $€$ & - & $€$ & $3.813,5$ & $€$ & 635,1 \\
\hline & 2 & $€$ & - & $€$ & $8.619,7$ & $€$ & - & $€$ & 658,8 \\
\hline & 3 & $€$ & - & $€$ & - & $€$ & $1.156,6$ & $€$ & - \\
\hline & 4 & $€$ & - & $€$ & $1.395,4$ & $€$ & - & $€$ & $2.254,5$ \\
\hline \multicolumn{2}{|c|}{$O C\left(G_{i}\right)[€]$} & $€$ & $3.879,3$ & $€$ & $10.015,1$ & $€$ & $4.970,1$ & $€$ & $3.548,4$ \\
\hline
\end{tabular}


OC has $n_{g}$ elements, as the number of orders. $O C_{i}$, instead, is the cost in manufacturing the $i-t h$ order with the proposed clustering in a certain number of builds $j$; the number of parts realized for each build is represented by $S_{i j}$.

$O C_{i}=\sum_{j}^{n_{b}} T P C_{i j} S_{i j} \forall i=1, n_{g}$.

Previously, the penalties for a delay in delivery were presented; the mathematical formulation of this cost is the following.

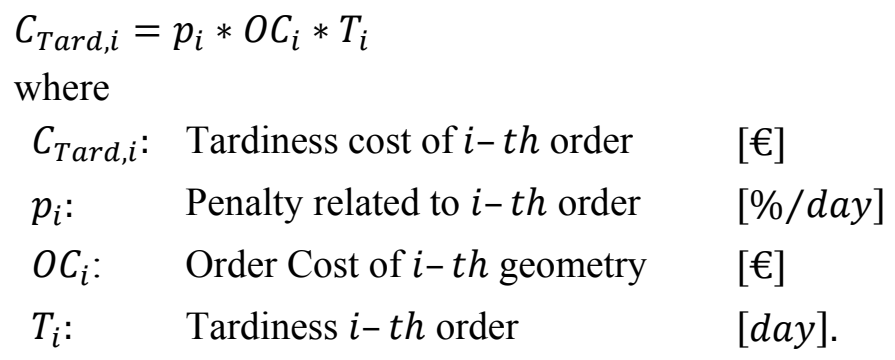

In the industrial sector, the cost of tardiness is proportional to the number of tardiness days. $O C_{i}$ increases of a quote $\left(p_{i}\right)$ for each day of tardiness. The sum of OC and tardiness cost for $i-t h$ geometry is defined as the Total Order Cost $\left(T O C_{i}\right)$ :

$T O C_{i}=O C_{i}+C_{\text {Tard }, i}$.

On the basis of the problem structure, the TOC mathematical formulation is presented as the following vector:

$$
T O C=\left[T O C_{1}, T O C_{2}, \ldots, T O C_{n g}\right] .
$$

The TOC is a vector where each element represents the amount of money needed to satisfy the overall demand, with the proposed order clustering. It is worth noting that it is possible to compute also a scalar value to represent the economic effort to produce some geometries when a specific scheduling $(S)$ is applied. This unique value is the sum of all of the TOCs. This value is obtained by summing all of the TOCs for all of the order/geometries to be produced in a specific time frame.

$$
\mathrm{C}_{\mathrm{P}} A M=\sum_{i}^{n g} T^{n} O C_{i}=39054.18[€] .
$$

To account for the cost part realized in the time execution for the specific part, the $\mathrm{C}_{\mathrm{P}}$ factor is introduced. This element must be a-dimensional and comparable to the time part. To achieve the objectives of summability and comparability, an algorithm for finding the proper weight for the AM cost is proposed. The desired weight must make a-dimensional $\mathrm{C}_{\mathrm{P}}$ but on the same order of magnitude as $F_{E T}$.

$$
F_{E T}(S) \sim o(10)^{1} \quad C_{P}(S) \sim o(10)^{4} \text { or further. }
$$

To gain either of the goals, as anticipated before, a small algorithm that will be inside the general optimization procedure is presented as follows:

a. Find $F_{E T}$ order of magnitude, indicated as $k_{1}$.

b. Compute $\mathrm{C}_{\mathrm{P}}$ as sum: $\sum T O C_{i}$.

c. Find $C_{P}$ order of magnitude, indicated as $k_{2}$.

d. Compute order of magnitude $k_{x}$ using the following equation:

Now, compute weight $\gamma_{S}$ :

$$
10^{\mathrm{k}_{1}}=\frac{10^{\mathrm{k}_{2}}}{10^{\mathrm{k}_{\mathrm{x}}}} \rightarrow k_{\mathrm{x}}=\mathrm{k}_{2}-\mathrm{k}_{1} \text {. }
$$




$$
\gamma_{S}=\frac{1}{10^{\left(k_{2}-k_{1}\right)}} \text {. }
$$

Finally, the MOP Cost Part has the following weighted formulation:

\subsection{Model constraints}

$$
F_{C P}=\sum_{i}^{n_{g}} \gamma_{S} T O C_{i}
$$

The first constraint presented in the model in the previous paragraph is the one related to the build chamber volume. When a planner schedules a DMLS ${ }^{\circledR}$, one of the first issues to be considered as practical constraint is the chamber volume of the machine. The maximum value available is computed as follows:

$$
V_{\text {chamber }}=L_{x} * L_{y} * L_{z}
$$

where

$\begin{array}{llr}L_{x} & \text { X-axis plate dimension } & {[\mathrm{mm}]} \\ L_{y} & \text { Y-axis plate dimension } & {[\mathrm{mm}]} \\ L_{z} & \text { build chamber height } & {[\mathrm{mm}]} \\ V_{\text {chamber }} & \text { Build chamber volume } & {\left[\mathrm{mm}^{3}\right] .}\end{array}$

Each PN has a proper geometrical volume, evaluable from computer aided design (CAD) data; nevertheless, to assemble a build, we must increase the PN volume to match some production needs, such as the following two:

- Part orientation: to confer precise mechanical characteristics to a product, the PN has to be built in selected growth directions. To ensure this growth direction, the designer should consider support structures that are needed for extra volume.

- Removal space: the planner must consider the necessary space for manual part removal, so the geometries in a build cannot be too close to each other, which produces another extra volume.

The planner gets the extra volume required for part orientation and manual removal directly from the designer and adds it to the PN geometrical volumes. This "global" volume info is simply referred to as "volume" for planning operations and saved in the form of a vector, as in the following:

$$
V=\left[V_{1}, V_{2}, \ldots, V_{n_{g}}\right]
$$

Once the elements of this constraint are presented, let us present the mathematical formula for the volume constraint.

$$
\sum_{i}^{n_{g}} n_{i, j} * V_{i} \leq V_{\text {chamber }} \forall j \in\left[1, n_{b}\right]
$$

where

$$
\begin{aligned}
& n_{i, j} \quad \text { Number item } i-t h \text { in } j-t h \text { [part] } \\
& \text { build } \\
& V_{i} \quad \text { Volume of } i-t h \text { geometry } \quad\left[\mathrm{cm}^{3}\right] \\
& V_{\text {chamber }} \text { Build chamber volume } \quad\left[\mathrm{cm}^{3}\right] \\
& n_{g} \quad \text { Number of order in the build [-] }
\end{aligned}
$$


$n_{b} \quad$ Number of build in the $[-]$.

schedule

This constraint not only must be valid for a single build, but also for each $j$ - th build in the schedule.

After the geometrical volume constraint, the production constraint will be presented. This constraint requires that the sum of all clustered orders for the $i-t h$ PN provides the corresponding $i-t h$ demand value in the analyzed period. This condition is presented mathematically as follows:

$$
\sum_{j=1}^{n b} n_{i, j}=d_{i}, \quad \forall i=1, n_{g} .
$$

It is worth noting that the values of the variable $n_{i, j}$ can be included in the following interval:

$$
n_{i, j} \in\left[0, n_{\max }\right] \text {. }
$$

This set of values has a lower limit always equal to 0 and an upper limit $n_{\max }$, which is continuously updated because it is connected to the $i-t h$ unit present in build $(j-1)-t h$. This condition can be summarized as follows:

$$
n_{\max }=\left\{\begin{array}{cc}
\bar{d}_{l}, & j=1 \\
\bar{d}_{\imath}-\sum_{j=1}^{j-1} n_{i, j}, & j>1
\end{array} .\right.
$$

The $\bar{d}_{l}$ value seems to recall the demand vector values for the $i-t h$ order, but in this vector, there is another element to take into account, which is directly connected to the volume constraint. In fact, it is important to remember that the machine chamber could not accomplish necessarily the total quantity $d_{i}$ because the volume constraint is present, so the value of demand assigned to the $j-t h$ build will be computed using the following formula, which is the product of the two model constraints in practice:

$$
\bar{d}_{\iota}=\min \left(d_{i},\left\lfloor\frac{V_{\text {chamber }}}{V_{i}}\right\rfloor\right) \text {. }
$$

Even if the last mathematical representation is the result of the two constraints mixed, we decided to use the representation of the previous paragraphs with the two separate constraints. This solution can be easier in the implementation of the present method in a real case.

\subsection{Computational complexity}

The proposed multi-objective model has two fundamental parts: one for the optimization of the E\&T, derived from the literature, and another proposed by the authors for cost optimization.

The E\&T weighted form is similar to following formulation:

$$
\sum_{j=1}^{n} \alpha E_{j}+\beta T_{j} \rightarrow \sum_{j=1}^{n} E_{j}+T_{j} .
$$

In fact, $\alpha$ and $\beta$ are constant weights, and they do not influence problem complexity.

The formulation on the right is NP-HARD, as presented by Wan and Yuan (2013). Therefore, adding the cost part to the E\&T formulation produces an increase of complexity, so it is possible to say that our problem is an NP-HARD computational problem. For this reason, a heuristics approach will be developed.

\section{Solver design}

Since the last part of the previous paragraph stated the NP-HARD complexity, as mentioned before in this paper, a heuristics method will be presented to solve the problem. In particular, because the theme is very new in the scheduling sector, we preferred to apply a very well-known heuristics, i.e., the genetic 
algorithm (GA). Nevertheless, we must have some considerations on the original GA configuration because the AM scheduling presents differences from the traditional single-machine scheduling problem. In fact, as we will present, these techniques are good in manipulating the schedule as a vector shape; in AMSP, there is a matrix, so we need to update the cited algorithms to use them on our proposed structure. Starting from this traditional approach, in this paper, a modified version of the Genetic Algorithm (PGA) is proposed, starting from an initial population where $N_{p}$ is the number of random schedules. Index $k$ goes from 1 to $N_{p}$, which is the number of members in the initial configuration. The proposed GA mixes the initial population order to favor diversification; later, parents $k$-th and $k+1-$ th $\left(P_{1}, P_{2}\right)$ intersect and produce two children $(\mathrm{C} 1, \mathrm{C} 2)$. Now, there is a set of four schedules represented by $P C=$ $\left\{P_{1}, P_{2}, C_{1}, C_{2}\right\}$, where PGA selects two schedules with the best OF value and replaces $P_{1}$ and $P_{2}$.

In the above set PC, better schedules could be any of the possible choices available in the set. For instance, the typical elements of the GA are presented in Fig. 4.

Generally, to mix the parents' genes, a crossover operator is applied, which goes on until the counter $k$ respects the relation $k<N_{p}$, i.e., when a new generation is fully completed. The overall termination criterion for all algorithms is generally the standard deviation $\sigma\left(F_{s}\right)$ of the OF over the entire population. When $\sigma\left(F_{S}\right)$ is less than an imposed tolerance $\left(10^{-6}\right)$, the algorithm terminates because all members are the same (similar).

Nevertheless, there is an emergency termination criterion about maximum no-improvement iteration number, because sometimes convergence condition requires too much time to be gained.

To let the readers better understand what will be proposed in this paper, the traditional crossover operators applied to the GA are briefly reported (Fig. 4). The core of GA is the so-called crossover mechanism that turns two parents in the same number of child. In a single-machine traditional scheduling problem, there is a sequence of jobs that represent a chromosome; instead, each job is a gene. Two feasible solutions are two chromosomes that must be intersected, by different crossover; some of them are

- Fixed cross-point;

- Random cross-point;

- Double cross-point.

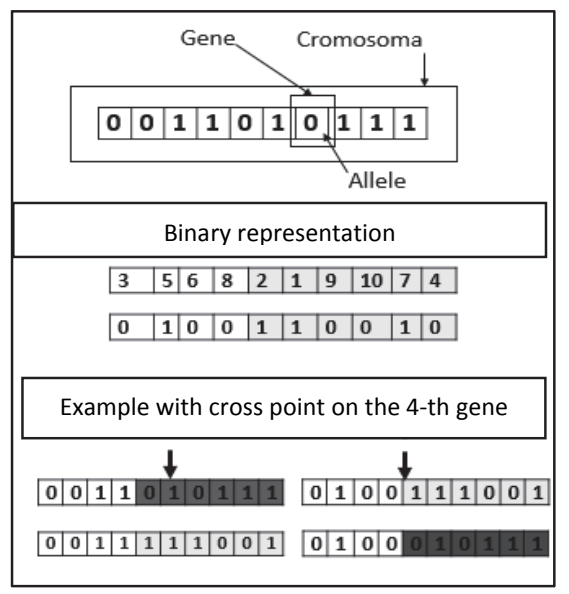

Fig. 4. Chromosome, genes, and crossover

The above cross-points are well suited to operate on a job sequence as a vector form, i.e., a 1-dimensional structure. In a single-machine AM scheduling problem, there is a matrix, so we must propose some changes. As anticipated before, an AM problem presents a 2D structure, and this suggests a 2D crossover 
(2DC) mechanism. The proposed 2DC provides a double intersection and operates as follows. At first, it considers orders as the genes and generates a random number called $i \epsilon\left[1, n_{g}-1\right]$. Random number $i$ is the column where cut schedule; this leads to two groups of chromosomes. In the following example of Table $5, i=2$. The first intersection is an exchange of the same color (green and blue) columns.

Once the first intersection is realized, we generate a second random number $j \epsilon\left[1, n_{b}-1\right]$. Random number $\mathrm{j}$ represents the row where cut schedule; this leads to two groups of chromosomes. In the following example of Table $6, j=3$. Thus, the second intersection is an exchange of the same color rows (green, red, blue orange). The bi-dimensional form of the problem also has connections and consequences on the resolution techniques.

\section{Test case}

In this section, the proposed model is tested using a test case. This experiment is useful in checking the performances of the model and the proposed algorithms. The proposed test case covers situations usually most critical for conventional manufacturing production systems, i.e., a production composed of different orders with the following characteristics: low volume, high geometrical variability, and great difficulty to gain Economic Production Quantity (EPQ).

\section{Table 5}

Horizontal intersection of 2D crossover

\begin{tabular}{|l|l|l|l|l|}
\hline 7 & 0 & 4 & 1 & 1 \\
\hline 0 & 7 & 0 & 1 & 1 \\
\hline 0 & 0 & 1 & 0 & 2 \\
\hline 0 & 1 & 0 & 4 & 1 \\
\hline
\end{tabular}

\begin{tabular}{|l|l|l|l|l|}
\hline 7 & 8 & 1 & 5 & 4 \\
\hline 0 & 0 & 1 & 1 & 0 \\
\hline 0 & 0 & 0 & 0 & 1 \\
\hline 0 & 0 & 3 & 0 & 0 \\
\hline
\end{tabular}

\begin{tabular}{|l|l|l|l|l|}
\hline 7 & 8 & 4 & 1 & 1 \\
\hline 0 & 0 & 0 & 1 & 1 \\
\hline 0 & 0 & 1 & 0 & 2 \\
\hline 0 & 0 & 0 & 4 & 1 \\
\hline
\end{tabular}

\begin{tabular}{|l|l|l|l|l|}
\hline 7 & 0 & 1 & 5 & 4 \\
\hline 0 & 7 & 1 & 1 & 0 \\
\hline 0 & 0 & 0 & 0 & 1 \\
\hline 0 & 1 & 3 & 0 & 0 \\
\hline
\end{tabular}

\section{Table 6}

Vertical intersection of 2D crossover

\begin{tabular}{|l|l|l|l|l|}
\hline 7 & 8 & 4 & 1 & 1 \\
\hline 0 & 0 & 0 & 1 & 1 \\
\hline 0 & 0 & 1 & 0 & 2 \\
\hline 0 & 0 & 0 & 4 & 1 \\
\hline
\end{tabular}

\begin{tabular}{|l|l|l|l|l|}
\hline 7 & 0 & 1 & 5 & 4 \\
\hline 0 & 7 & 1 & 1 & 0 \\
\hline 0 & 0 & 0 & 0 & 1 \\
\hline 0 & 1 & 3 & 0 & 0 \\
\hline
\end{tabular}

\begin{tabular}{|l|l|l|l|l|}
\hline 0 & 1 & 3 & 0 & 0 \\
\hline 7 & 0 & 1 & 5 & 4 \\
0 & 7 & 1 & 1 & 0 \\
\hline 0 & 0 & 0 & 0 & 1 \\
\hline
\end{tabular}

\begin{tabular}{|l|l|l|l|l|}
\hline 0 & 0 & 0 & 4 & 1 \\
\hline 7 & 8 & 4 & 1 & 1 \\
\hline 0 & 0 & 0 & 1 & 1 \\
\hline 0 & 0 & 1 & 0 & 2 \\
\hline
\end{tabular}

$F_{s}=7,80$

First, from this experiment, we expect that, starting from one or a family of feasible solutions, PGA is able to optimize them, reducing the OF value. This experimental result will ensure, at least, the computational effectiveness of the proposed techniques, while its effectiveness when compared with other heuristics is not tested since it is a new problem not yet faced from the scientific community and the comparison with other heuristics will be tested I future steps of the research.

In the second step of the experiment, we will discuss the algorithms efficiency, looking in particular to

- due date respect that will be measured from the traditional service level index;

- cost reduction that will be measured in percentage of the basic value;

- running time that will be measured in seconds.

\subsection{Test case data set}

Test case data are shown in Table 7. There are 30 PNs, highly different in form and dimension, but with low production volume, between 5 and 10 units. Production Orders for the AM department cover six 


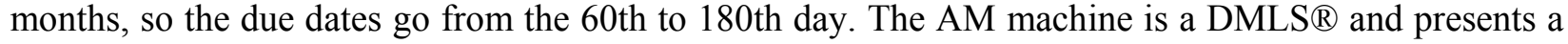
build chamber volume of $V_{\max }=13437.50 \mathrm{~cm}^{3}$.

The experiments were performed using a laptop equipped with Intel Core $i 7 \AA-4700 \mathrm{MQ}, 2.40 \mathrm{GHz}$ CPU, and 16 GB RAM. All algorithms are coded in Matlab ${ }^{\circledR} 7$ R2015a.

Table 7

Test case data

\begin{tabular}{|c|c|c|c|c|c|c|c|c|}
\hline PN [\#] & $\mathrm{DD}[d a y]$ & $\mathrm{D}$ [pieces] & $\mathrm{V}\left[\mathrm{cm}^{3}\right]$ & $\mathrm{h}[\mathrm{mm}]$ & $\rho\left[\mathrm{g} / \mathrm{cm}^{3}\right]$ & $\mathrm{T}_{\text {prep }}[$ hours $]$ & $\begin{array}{l}\text { Penalty } \\
{[\% / d a y]}\end{array}$ & $\mathrm{S}_{\max }\left[\mathrm{cm}^{2}\right]$ \\
\hline 1 & 120 & 7 & 146 & 50,55 & 8 & 1 & 1 & 82,1 \\
\hline 2 & 60 & 5 & 52,87 & 85 & 8 & 1 & 2 & 120 \\
\hline 3 & 180 & 10 & 108,9 & 62,5 & 8 & 1 & 2 & 344,22 \\
\hline 4 & 120 & 7 & 64,17 & 37,73 & 8 & 1 & 1,5 & 21 \\
\hline 5 & 120 & 5 & 200,8 & 183,4 & 8 & 0,5 & 3 & 208,08 \\
\hline 6 & 90 & 5 & 66,94 & 56,02 & 8 & 1 & 1,5 & 178,72 \\
\hline 7 & 120 & 5 & 90,15 & 95 & 8 & 0,5 & 2 & 57,04 \\
\hline 8 & 120 & 8 & 188,2 & 162,5 & 8 & 1 & 2 & 104,56 \\
\hline 9 & 150 & 9 & 33,65 & 32,29 & 8 & 1 & 1,5 & 97,12 \\
\hline 10 & 180 & 9 & 290,2 & 186,6 & 8 & 1 & 1,5 & 112,28 \\
\hline 11 & 60 & 5 & 62 & 150 & 8 & 0,5 & 1 & 176,715 \\
\hline 12 & 180 & 10 & 6 & 73 & 8 & 1 & 2 & 41,8539 \\
\hline 13 & 180 & 10 & 9 & 65 & 8 & 0,4 & 1 & 33,1831 \\
\hline 14 & 90 & 8 & 56 & 115 & 8 & 0,6 & 2 & 103,869 \\
\hline 15 & 120 & 5 & 17 & 100 & 8 & 0,4 & 1 & 213,825 \\
\hline 16 & 150 & 8 & 44 & 165 & 8 & 0,4 & 3 & 78,5389 \\
\hline 17 & 120 & 7 & 4,87 & 100 & 8 & 0,1 & 1 & 3,14159 \\
\hline 18 & 60 & 5 & 2,9 & 22 & 8 & 0,2 & 1 & 38,4845 \\
\hline 19 & 180 & 10 & 112 & 70 & 8 & 0,4 & 1 & 116,899 \\
\hline 20 & 90 & 5 & 150 & 122 & 8 & 0,4 & 2 & 201,062 \\
\hline 21 & 150 & 8 & 375 & 160 & 8 & 0,7 & 1 & 4,90874 \\
\hline 22 & 60 & 5 & 17,5 & 25 & 8 & 0,1 & 1 & 28,2743 \\
\hline 23 & 90 & 6 & 36 & 60 & 8 & 0,2 & 1 & 12,5664 \\
\hline 24 & 120 & 7 & 13,4 & 40 & 8 & 0,2 & 1 & 10,1788 \\
\hline 25 & 90 & 5 & 22,6 & 36 & 8 & 0,3 & 2 & 9,62113 \\
\hline 26 & 120 & 7 & 7 & 35 & 8 & 0,4 & 2 & 12,5664 \\
\hline 27 & 60 & 4 & 11 & 40 & 8 & 0,4 & 1 & 10,1786 \\
\hline 28 & 90 & 5 & 4 & 45 & 8 & 0,4 & 2 & 9,62113 \\
\hline 29 & 120 & 7 & 15 & 50 & 8 & 0,4 & 2 & 19,635 \\
\hline 30 & 180 & 10 & 0,569 & 20 & 8 & 0,4 & 2 & 3,14159 \\
\hline
\end{tabular}

\subsection{Initial Solution Algorithm (ISA)}

As cited in Section 4, the production orders are the only information required for the implementation of the model. The AM schedule that satisfies the demand is a matrix with a fixed column number, but with a variable number of rows (builds). This is due to the order clustering principle. A first feasible solution is needed, and this solution is developed by a third algorithm, named ISA, to gain an Initial Random Schedule, as shown in Table 8 . The ISA algorithm can produce one or a family of $N$ feasible solutions.

\section{Table 8}

Set of 10 possible initial feasible solutions

\begin{tabular}{|c|c|c|c|c|c|c|c|c|}
\hline $\mathrm{S}$ & [\#] & $\mathrm{F}_{\mathrm{ET}}$ & $\mathrm{F}_{\mathrm{COST}}$ & {$[-]$} & N BUILD [\#] & {$[\mathrm{k} €]$} & {$[\%]$} & $\mathrm{C}_{\text {tardiness }}[k \epsilon]$ \\
\hline & 1 & 11,44 & 17,52 & 28,96 & 9 & 175,20 & 43,33 & 36,31 \\
\hline & 2 & 12,62 & 17,20 & 29,82 & 7 & 172,00 & 43,33 & 33,12 \\
\hline & 3 & 14,30 & 17,64 & 31,94 & 10 & 176,39 & 40,00 & 35,04 \\
\hline & 4 & 13,78 & 17,69 & 31,47 & 7 & 176,92 & 30,00 & 39,89 \\
\hline & 5 & 11,41 & 16,10 & 27,50 & 10 & 160,98 & 46,67 & 19,36 \\
\hline & 6 & 12,36 & 16,68 & 29,04 & 9 & 166,79 & 50,00 & 27,61 \\
\hline & 7 & 11,75 & 17,02 & 28,77 & 9 & 170,18 & 43,33 & 28,84 \\
\hline & 8 & 15,25 & 16,36 & 31,61 & 8 & 163,59 & 40,00 & 24,51 \\
\hline & 9 & 10,63 & 16,53 & 27,16 & 8 & 165,30 & 46,67 & 26,82 \\
\hline & 10 & 14,66 & 17,80 & 32,45 & 8 & 177,96 & 30,00 & 39,51 \\
\hline
\end{tabular}


Moreover, as an input, there is a parameter $0<k \leq 1$ capable of modifying the maximum chamber volume:

$$
V_{\text {available }}=k * V_{\text {max }}
$$

This parameter alters the volume filling, expanding the feasible solution region and metaheuristics searching domain. This simple algorithm generates a first random vector of integers, using randi() $M a t l a b^{\circledR}$ function, after the algorithm verifies production and volume constraints. If the build is feasible and partially empty, an Optimization Module starts to fill it with the PNs with sooner due dates. The random build generation, as well as $k$ parameter, contributes to the diversification of the solutions. The initial feasible population is shown in Table 8 .

\subsection{PGA implementation and results}

The PGA works with a random point 2D crossover (as described before), so it is normal to observe different results after different trials, as shown in Table 9.

Table 9

Proposed Genetic Algorithm results

\begin{tabular}{ccccccc}
\hline & & \multicolumn{5}{c}{ Run [\#] } \\
\cline { 3 - 7 } & & $\mathbf{1}$ & $\mathbf{2}$ & $\mathbf{3}$ & $\mathbf{4}$ & $\mathbf{5}$ \\
\hline OF & {$[-]$} & 6.60 & 6.05 & 7.92 & 7.60 & 7.42 \\
CP & {$[\mathrm{k} €]$} & 153.12 & 149.02 & 157.73 & 155.24 & 156.71 \\
$\mathbf{L S}$ & {$[\%]$} & 56.67 & 60.00 & 56.67 & 66.67 & 71.43 \\
$\mathbf{I t}$ & {$[\#]$} & 1126 & 1119 & 524 & 1122 & 1122 \\
\hline
\end{tabular}

Table 10

Results of Genetic Algorithm

\begin{tabular}{ccccc}
\hline & & Initial & Final & $\Delta[\%]$ \\
\hline OF & {$[-]$} & 27.16 & 6.05 & -77.72 \\
CP & {$[\mathbf{k €}]$} & 165.30 & 149.02 & -9.85 \\
LS & {$[\%]$} & 46.67 & 60.00 & 28.57 \\
\hline
\end{tabular}

Generally, PGA gives good solutions, with a sensible reduction of OF. The iteration number is greater than 1000 in all cases, but less than in \#3 (it $=524$ ), which also presents the worst result among trials. Table explains the details of case \#2, the best result among the five trials performed. As shown in Table 10 , the OF improvement is greater than $77 \%$, the cost of production decreases by $9.85 \%$, and the LS increases by $28.57 \%$.

\section{Conclusions and discussion of the results}

In this paper, a new research theme related to operation management optimization using a single machine with the AM technology was discussed. In particular, the mathematical problem was stated as a multiobjective function based on the balancing of the optimization of earliness/tardiness and production costs (considering also the penalties for delayed orders). This multi-objective function has two main constraints: one for the geometrical volume of the printing chamber and one for the respect of the due dates. Built the mathematical model (that is a NP-HARD problem) in this work it was presented a traditional heuristic, modified to respect the AM technological characteristics, i.e., the Genetic Algorithms. This algorithm was applied to a test case built for the occasion, which simulates the case in which a traditional manufacturing production system receives orders that generally make difficulties in the respect of the demand, i.e., orders characterized by low volumes and high geometrical variability between the PNs to be realized. The heuristics were applied, and the optimized results from the first initial solution were produced using a simple algorithm illustrated before, named ISA.

Therefore, it is possible to say that the single-machine problem using the AM technology can be solved using a heuristic such as the PGA, which was modified for the occasion, having very good results in terms of operations and very good calculation running time. In fact, we can see that PGA has good performances in all of the three evaluation parameters, i.e., the value of the objective function, the value of production costs, and the service level percentage, including the calculation time. In a future work, we 
will compare the heuristics presented here with others such as the tabu search or the particle swarm optimization.

\section{References}

Costabile, G., Fera, M., Fruggiero, F., Lambiase, A., \& Pham, D. (2017). Cost models of additive manufacturing: A literature review. International Journal of Industrial Engineering Computations, 8(2), 263-283.

Dilberoglu, U. M., Gharehpapagh, B., Yaman, U., \& Dolen, M. (2017). The role of additive manufacturing in the era of industry 4.0. Procedia Manufacturing, 11, 545-554.

Fera, M., Fruggiero, F., Lambiase, A., \& Macchiaroli, R. (2016a). State of the art of additive manufacturing: Review for tolerances, mechanical resistance and production costs. Cogent Engineering, 3(1), 1261503.

Fera, M., Macchiaroli, R., Fruggiero, F. \& Lambiase, A. (2017). A new perspective for production process analysis using additive manufacturing - complexity vs production volume. International Journal of Advanced Manufacturing and Technology, https://doi.org/10.1007/s00170-017-1221-1

Fera, M., Macchiaroli, R., Iannone, R., Miranda, S., \& Riemma, S. (2016b). Economic evaluation model for the energy Demand Response. Energy, 112, 457-468.

Fruggiero, F., Riemma, S., Ouazene, Y., Macchiaroli, R., \& Guglielmi, V. (2016). Incorporating the human factor within manufacturing dynamics. IFAC-PapersOnLine, 49(12), 1691-1696.

Fera, M., Costabile, G., Fruggiero, F., Lambiase, A., \& Pham, D. T., (2017). A new mixed production cost allocation model for additive manufacturing (MiProCAMAM). International Journal of Advanced Manufacturing Technology, 92(9-12), 42754291.

Jin, Y., Du, J., \& He, Y. (2017). Optimization of process planning for reducing material consumption in additive manufacturing. Journal of Manufacturing Systems, 44, 65-78.

Li, Q., Kucukkoc, I., \& Zhang, D. Z. (2017). Production planning in additive manufacturing and 3D printing. Computers \& Operations Research, 83, 157-172.

Nearchou, A. C. (2010). Scheduling with controllable processing times and compression costs using populationbased heuristics. International Journal of Production Research, 48(23), 7043-7062.

Newman, S. T., Zhu, Z., Dhokia, V., \& Shokrani, A. (2015). Process planning for additive and subtractive manufacturing technologies. CIRP Annals-Manufacturing Technology, 64(1), 467-470.

Pour, M. A., Zanardini, M., Bacchetti, A., \& Zanoni, S. (2016). Additive Manufacturing Impacts on Productions and Logistics Systems. IFAC-PapersOnLine, 49(12), 1679-1684.

Ransikarbum, K., Ha, S., Ma, J., \& Kim, N. (2017). Multi-objective optimization analysis for part-to-Printer assignment in a network of 3D fused deposition modeling. Journal of Manufacturing Systems, 43, 35-46.

Ren, L., Sparks, T., Ruan, J., \& Liou, F. (2008). Process planning strategies for solid freeform fabrication of metal parts. Journal of Manufacturing Systems, 27(4), 158-165.

Wan, L., \& Yuan, J. (2013). Single-machine scheduling to minimize the total earliness and tardiness is strongly NP-hard. Operations Research Letters, 41(4), 363-365.

Witherell, P., Lu, Y., \& Jones, A. (2017). Additive manufacturing: A trans-disciplinary experience. In Transdisciplinary Perspectives on Complex Systems (pp. 145-175). Springer International Publishing.

Zhang, Y., Gupta, R. K., \& Bernard, A. (2016). Two-dimensional placement optimization for multi-parts production in additive manufacturing. Robotics and Computer-Integrated Manufacturing, 38, 102-117.

Zhu, Z., Dhokia, V., \& Newman, S. T. (2017). A novel decision-making logic for hybrid manufacture of prismatic components based on existing parts. Journal of Intelligent Manufacturing, 28(1), 131-148.

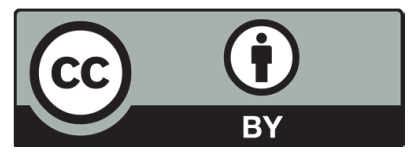

(C) 2018 by the authors; licensee Growing Science, Canada. This is an open access article distributed under the terms and conditions of the Creative Commons Attribution (CCBY) license (http://creativecommons.org/licenses/by/4.0/). 